\title{
Analytical expressions for group delay in the far field from an optical fiber having an arbitrary index profile
}

\author{
Danielsen, Per Lander
}

Published in:

I E E E Journal of Quantum Electronics

Link to article, DOI:

10.1109/JQE.1981.1071223

Publication date:

1981

Document Version

Publisher's PDF, also known as Version of record

Link back to DTU Orbit

Citation (APA):

Danielsen, P. L. (1981). Analytical expressions for group delay in the far field from an optical fiber having an arbitrary index profile. I E E E Journal of Quantum Electronics, 17(6), 850-853.

https://doi.org/10.1109/JQE.1981.1071223

\section{General rights}

Copyright and moral rights for the publications made accessible in the public portal are retained by the authors and/or other copyright owners and it is a condition of accessing publications that users recognise and abide by the legal requirements associated with these rights.

- Users may download and print one copy of any publication from the public portal for the purpose of private study or research.

- You may not further distribute the material or use it for any profit-making activity or commercial gain

- You may freely distribute the URL identifying the publication in the public portal

If you believe that this document breaches copyright please contact us providing details, and we will remove access to the work immediately and investigate your claim. 


\title{
Analytical Expressions for Group Delay in the Far Field from an Optical Fiber Having an Arbitrary Index Profile
}

\author{
PER L. DANIELSEN
}

\begin{abstract}
A general and efficient model for optical fibers with a few modes and arbitrary index profiles is established. The model yields a solution of the vectorial wave equation and analytical expressions for the group delay and the far field. Convergence tests have shown that the dispersion can be calculated with an accuracy better than 0.2 $\mathrm{ps} /(\mathrm{km} \cdot \mathrm{nm})$.
\end{abstract}

\section{INTRODUCTION}

I $\mathrm{N}$ a single-mode fiber only one mode can propagate the $L P_{01}$ mode. The reason for including more $L P$ modes in the investigation is that the investigation then includes an alternative to the single-mode fiber-the dual-mode fiber [1]. In a dual-mode fiber, the $\mathrm{LP}_{01}$ and $\mathrm{LP}_{11}$ modes can propagate. Furthermore, measurement of the pulse response in the two LP-mode regions and measurement of the far field in the single-mode region can be used to determine the index profile [2].

When investigating the fiber at wavelengths near the cutoff of the first higher order mode, care has to be taken in the calculation of the group delay of the higher order mode. This means that the vectorial wave equation has to be used instead of the scalar wave equation. The result of using the vectorial wave equation is that the $\mathrm{LP}_{11}$ mode separates into the $\mathrm{HE}_{21}$ and $\mathrm{EH}_{01}$ modes, which have different group delay close to cutoff [2].

In this paper, we shall discuss the calculation of the propagation constant, the group delay, the dispersion, and the far field. The calculations will be based on the vectorial wave equation and the formulas will be valid for every mode that can propagate. Because the index profile is sampled in a finite number of points, it is not possible to rapidly investigate varying profiles without the use of a great number of points.

The method used is an approximation of the index profile by a multilayer structure where each layer has a constant refractive index. It is faster than some earlier methods of solving the vectorial wave equation by use of a multilayer structure [3], [5] because it only deals with $2 \times 2$ matrices. Contrary to another multilayer method [4] dealing with $2 \times 2$ matrices, the present method can also be used on a $w$-profile because it includes a check of the field, i.e., if it really is evanescent far from the core. In the earlier method, it was assumed that the

Manuscript received November 20, 1980; revised January 12, 1981. This work was supported in part by the Danish Council for Scientific and Industrial Research.

The author is with the Electromagnetics Institute, the Technical University of Denmark, Lyngby, Denmark. field was evanescent far from the core, which introduces nonphysical modes in a $w$-profile. The electromagnetic field of these nonphysical modes is only zero at the boundary between the cladding and the outermost layer with a high refractive index, but increases rapidly in the cladding. As mentioned earlier, we need a very accurate calculation of the group delay close to cutoff, and it can be obtained by an analytical integration of the field. This is discussed in Sections III and IV. In Section V, a formula is derived giving the far field from an arbitrary mode.

\section{The Characteristic Equation}

The electromagnetic field $\bar{E}$ in any circular waveguide symmetric about the $z$-axis can be expressed as a superposition of three orthogonal vector fields:

$$
\bar{E}=\bar{E}^{+}+\bar{E}^{-}+E_{z} \cdot \hat{z} .
$$

$\bar{E}^{+}$and $\bar{E}^{-}$represent two vectors rotating counter and clockwise about the $z$-axis. $E_{z}$ is the $z$-component of the field and $\hat{z}$ is a unity vector along the $z$-axis. $\bar{E}^{+}$and $\bar{E}^{-}$can be decomposed in the following manner:

$$
\bar{E}^{ \pm}(r, \phi, z)=E^{ \pm}(r, \phi)(\hat{x} \mp j \hat{y}) e^{j(\omega t-\beta z)}
$$

where $r$ and $\phi$ are the polar coordinates, $\hat{x}$ and $\hat{y}$ are unity vectors, $\omega$ is the angular frequency, $t$ is the time, and $\beta$ is the propagation constant. The magnetic field $\bar{H}$ obeys a similar superposition. Furthermore, it is convenient to consider the following linear combinations of the axial components:

$$
\Phi_{z}^{ \pm}=j E_{z} \pm Z H_{z}
$$

where $Z$ is the characteristic impedance [4].

The electrical field can be separated into a radial and an angular part

$$
E^{ \pm}(r, \phi)=E^{ \pm}(r) e^{j m \phi}
$$

where $m$ is a nonzero integer. Insertion of (1) and (2) in the vectorial wave equation shows that the $r$-dependent part of $E^{ \pm}$satisfies the Bessel differential equation if the refractive index is constant [4].

We thus divide the index distribution into a multilayer structure with $N$ layers of constant refractive indexes $n_{i}$; see Fig. 1. Layer $N$ extends from radius equal to $a_{N-1}$ to infinity. Note that it is not necessary to require that the layers have the same thickness.

In [4] it is shown that $\Phi_{z}^{ \pm}$are related to $E^{ \pm}$through the 


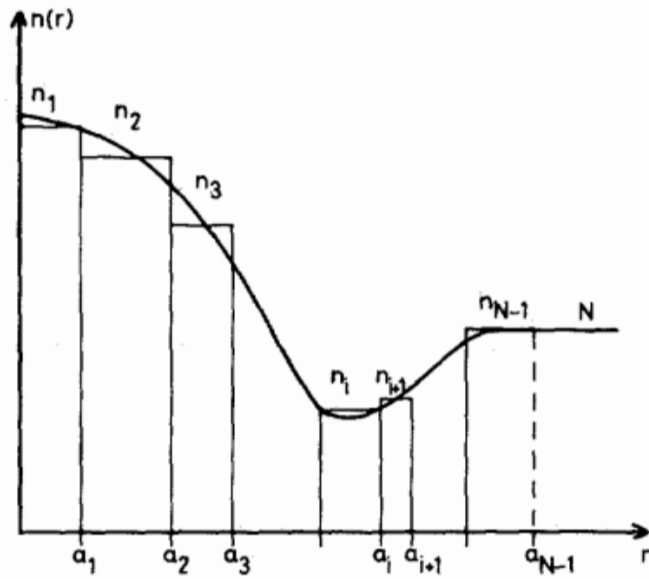

Fig. 1. The refractive index $n$ as function of radius $r$ and the multilayer approximation.

setup and the setdown operator for the Bessel functions. This means that the solutions in layer $i$ are, in a matrix formulation,

$$
\left(\begin{array}{c}
\Phi_{z}^{ \pm}(r) \\
E^{ \pm}(r)
\end{array}\right)_{i}=\overline{\bar{h}}_{i}^{ \pm}(r)\left(\begin{array}{c}
A_{i}^{ \pm} \\
B_{i}^{ \pm}
\end{array}\right) .
$$

The constants $A_{i}^{ \pm}$and $B_{i}^{ \pm}$depend on the layer $i$, the sign, and $m$. The matrix $\bar{h}_{i}$ is given by

$$
\overline{\bar{h}}_{i}^{ \pm}(r)=\left(\begin{array}{ll}
\frac{\kappa_{i}}{\beta} J_{m}\left(\kappa_{i} r\right) & \pm \frac{\kappa_{i}}{\beta} N_{m}\left(\kappa_{i} r\right) \\
J_{m \pm 1}\left(\kappa_{i} r\right) & N_{m \pm 1}\left(\kappa_{i} r\right)
\end{array}\right)
$$

for $\beta<k n_{i}$, where $k$ is the free space wavenumber. $J_{m}$ and $N_{m}$ are the Bessel and Neumann functions of order $m$ and

$$
\kappa_{i}=\sqrt{\left(k n_{i}\right)^{2}-\beta^{2}} \text {. }
$$

For $\beta>k n_{i}$, we have instead

$$
\overline{\bar{h}}_{i}^{ \pm}(r)=\left(\begin{array}{cc}
\frac{\gamma_{i}}{\beta} I_{m}\left(\gamma_{i} r\right) & -\frac{\gamma_{i}}{\beta} K_{m}\left(\gamma_{i} r\right) \\
I_{m \pm 1}\left(\gamma_{i} r\right) & K_{m \pm 1}\left(\gamma_{i} r\right)
\end{array}\right)
$$

where $K_{m}$ and $I_{m}$ are the modified Bessel functions of first and second kind and order $m$, and

$$
\gamma_{i}=\sqrt{\beta^{2}-\left(k n_{i}\right)^{2}} \text {. }
$$

Matching of the fields at the boundary between individual layers can be expressed by using the $t$-matrix

$$
\overline{\bar{h}}_{i+1}^{ \pm}\left(a_{i}\right)\left(\begin{array}{c}
A_{i+1}^{ \pm} \\
B_{i+1}^{ \pm}
\end{array}\right)=\overline{\bar{t}}_{i} \overline{\bar{h}}_{i}^{ \pm}\left(a_{i}\right)\left(\begin{array}{c}
A_{i}^{ \pm} \\
B_{i}^{ \pm}
\end{array}\right)
$$

if a relation between $E^{+}$and $E^{-}$is neglected, reducing the order of the matrices from 4 to 2 [4]. The $t$-matrix is then given by

$$
\overline{\overline{t_{i}}}=\left(\begin{array}{ll}
1 & 0 \\
0 & 1+\Delta_{i}
\end{array}\right)
$$

where

$$
\Delta_{i}=\frac{n_{i}^{2}-n_{i+1}^{2}}{2 n_{i}^{2}} .
$$

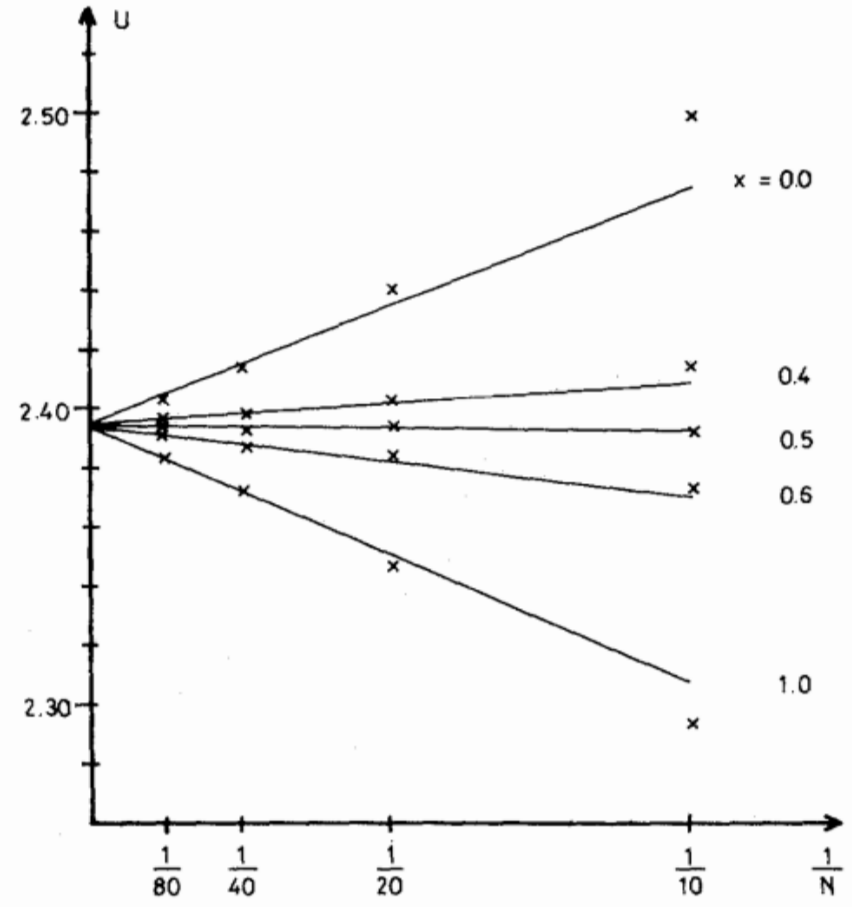

Fig. 2. The eigenvalue $U$ as function of the number of layers $N$, with the sampling parameter $x$ as parameter. The normalized frequency $V[13]$ is 3 and the index profile is an $\alpha$-profile [2] with $\alpha$ as 2 .

By using the fact that the field has to be finite at $r=0$, we have to choose $B_{1}^{ \pm}=0$ and arbitrarily set $A_{1}^{ \pm}=1$. The systems of linear equations [see (10)] can now be solved successively for $A_{i+1}^{ \pm}$and $B_{i+1}^{ \pm}$, starting with $i=1$. A characteristic equation is obtained by using the fact that the field also has to be finite for $r=\infty$, i.e., the characteristic equation is

$$
A_{N}^{ \pm}(\beta)=0 .
$$

The characteristic equation (13) has to be solved numerically and the solutions corresponding to the minus sign are $\mathrm{HE}$ modes and to the plus sign are $\mathrm{EH}$ modes [4]. The $\mathrm{HE}_{m+1, n}$ modes and the $\mathrm{EH}_{m-1, n}$ modes are nearly degenerated, except for the $\mathrm{HE}_{1, n}$ modes which have no counterparts. Even though the root finding in (13) needs a lot of evaluations of Bessel functions, the number of Bessel functions in the characteristic equation (13) is not less when the scalar wave equation is used [6].

One way of decreasing the computational work is to choose the optimal sampling of the index profile. We thus introduce the parameter $x$ through

$$
n_{i}=n\left(a_{i-1}\right)+x\left(n\left(a_{i}\right)-n\left(a_{i-1}\right)\right), \quad 0 \leqslant x \leqslant 1 .
$$

Fig. 2 shows the eigenvalue

$$
U=\sqrt{\left(k n_{\text {core }}\right)^{2}-\beta^{2}}
$$

for a parabolic index profile with the $x$-value as a parameter, as a function of the number of layers. It is observed that when the number of layers increases, $U$ converges to the same value. When $x=0.5, U$ is almost independent of $N$ and 10 layers are therefore sufficient. A physical explanation of why $U$ is independent of $N$ is that the areas under the true index profile and the staircase profile are nearly equal. 


\section{CONFINEMENT FACTORS}

The power per unit area transmitted in the $z$-direction is

$$
S_{z}=\frac{1}{2} \operatorname{Re}\left(\bar{E} \times \bar{H}^{*}\right) \hat{z}
$$

where Re represents "the real part of." By using the relation between $E^{ \pm}$and $H^{ \pm}$[7], it can be shown that the power transmitted in the $z$-direction in each mode is

$$
P^{ \pm}=\int_{0}^{\infty}\left|E^{ \pm}\right|^{2} r d r
$$

where some proportionality factors, including the $\phi$ integration, are omitted. The confinement factors are defined as the power transmitted in each layer, when the total power is unity, i.e.,

$$
\Gamma_{i}^{ \pm}=\frac{1}{P^{ \pm}} \int_{a_{i-1}}^{a_{i}}\left|E^{ \pm}\right|^{2} r d r .
$$

An advantage of solving the characteristic equation (13), instead of the characteristic equation in [4], is that we now know the constants $A_{i}^{ \pm}$and $B_{i}^{ \pm}$, and thus have an analytical expression for $\left|E^{ \pm}\right|$. The integrations in (18) can be performed analytically [8] and we will now use the result to calculate the group delay and dispersion.

\section{Group Delay and Dispersion}

The group delay per unit length $\tau$ is defined as

$$
\tau=-\frac{\lambda^{2}}{2 \pi c} \frac{d \beta}{d \lambda}
$$

where $c$ is the vacuum speed of light. A disadvantage of using (19) directly is that calculation of $\tau$ at one wavelength involves the solution of the characteristic equation at two wavelengths. The calculation of $\tau$ can also be performed analytically from the knowledge of the propagation constant at only one wavelength $\lambda[9]$ :

$$
\tau=\frac{k}{c \beta P^{ \pm}} \int_{0}^{\infty} n(r, \lambda) N(r, \lambda)\left|E^{ \pm}\right|^{2} r d r
$$

where the group index

$$
N=\frac{d(n k)}{d k} .
$$

For the refractive index a Sellmeier fit [10] can be used. This means that the differentiation in (21) can also be performed analytically.

Insertion of (18) in (21) gives, for the multilayer structure,

$$
\tau=\frac{k}{c \beta} \sum_{i=1}^{N} n_{i} N_{i} \Gamma_{i}^{ \pm}
$$

where $N_{i}$ is the group index in layer $i$. Figures similar to Fig. 2 are obtained for the group delay which is, like the eigenvalue $U$, much more dependent on the sampling parameter $x$ than on the number of layers.

The dispersion

$$
T=-\frac{d \tau}{d \lambda}
$$

can be found directly from numerical differentiation of (22), but here a numerical differentiation is not as critical as in (19). By increasing the number of layers in a parabolic profile to 20,40 , and 80 , the agreement between results is within 0.2 $\mathrm{ps} /(\mathrm{km} \cdot \mathrm{nm})$ when $x=0.5$. For other values of $x$, figures similar to Fig. 2 are again obtained. Furthermore, the results are in excellent agreement with results obtained in [11].

\section{FAR FIELD}

The far field may be obtained from the Frauenhofer diffraction integral [12]

$$
\begin{aligned}
E_{f}(r, \theta, \phi)= & \frac{j}{\lambda r} e^{-j k r} \int_{0}^{\infty} \int_{0}^{2 \pi} E_{0}\left(r_{0}, \phi_{0}\right) \\
& \cdot e^{j k r_{0} \sin \theta \cos \left(\phi-\phi_{0}\right)} r_{0} d \phi_{0} d r_{0}
\end{aligned}
$$

where $(r, \theta, \phi)$ is the usual spherical coordinates and $E_{0}\left(r_{0}\right.$, $\phi_{0}$ ) is the field at the fiber end. In the following we will use the scalar LP modes [13] because we are not interested in a possible small difference in group delay. We thus set $m \pm 1=l$ and get

$$
E_{0}=\left[A_{i} J_{l}\left(\kappa_{i} r_{0}\right)+B_{i} N_{l}\left(\kappa_{i} r_{0}\right)\right] \cos l \phi_{0}
$$

for $k n_{i}>\beta$ and

$$
E_{0}=\left[A_{i} I_{l}\left(\gamma_{i} r_{0}\right)+B_{i} K_{l}\left(\gamma_{i} r_{0}\right)\right] \cos l \phi_{0}
$$

for $k n_{i}<\beta$. Equations (25) and (26) are only valid for small angles $\theta$. For angles greater than about $10^{\circ}$ an obliquity factor has to be introduced. The angular integration in (24) is an integral representation of the Bessel function [14] and the remaining integral can thus be calculated analytically [15]. The result is, if some constants depending only on $r$ have been neglected,

$$
E_{f}=\left(\sum_{\kappa_{i}}+\sum_{\gamma_{i}}\right) \cos l \phi .
$$

The first summation

$$
\begin{aligned}
\sum_{\kappa_{i}}= & \sum_{i}^{\prime}\left[\frac { 1 } { \kappa _ { i } ^ { 2 } - \eta ^ { 2 } } \left\{A_{i} \mid r_{0}\left(\kappa_{i} J_{l+1}\left(\kappa_{i} r_{0}\right) J_{l}\left(\eta r_{0}\right)\right.\right.\right. \\
& \left.-\eta J_{l}\left(\kappa_{i} r_{0}\right) J_{l+1}\left(\eta r_{0}\right)\right)\left.\right|_{r_{0}=a_{i-1}} ^{r_{0}=a_{i}} \\
& +B_{i} \mid r_{0}\left(\kappa_{i} N_{l+1}\left(\kappa_{i} r_{0}\right) J_{l}\left(\eta r_{0}\right)\right. \\
& \left.\left.\left.-\eta N_{l}\left(\kappa_{i} r_{0}\right) J_{l+1}\left(\eta r_{0}\right)\right)\left.\right|_{r_{0}=a_{i-1}} ^{r_{0}=a_{i}}\right\}\right]
\end{aligned}
$$

is a summation of the layers where $k n_{i}>\beta$ and the second summation

$$
\begin{aligned}
\sum_{\gamma_{i}}= & \sum_{i}^{\prime \prime}\left[\frac { 1 } { \gamma _ { i } ^ { 2 } + \eta ^ { 2 } } \left\{A_{i} \mid r_{0}\left(\gamma_{i} K_{l+1}\left(\gamma_{i} r_{0}\right) J_{l}\left(\eta r_{0}\right)\right.\right.\right. \\
& \left.-\eta K_{l}\left(\gamma_{i} r_{0}\right) J_{l+1}\left(\eta r_{0}\right)\right)\left.\right|_{r_{0}=a_{i-1}} ^{r_{0}=a_{i}} \\
& +B_{i} \mid r_{0}\left(\gamma_{i} I_{l+1}\left(\gamma_{i} r_{0}\right) J_{l}\left(\eta r_{0}\right)\right. \\
& \left.\left.\left.-\eta I_{l}\left(\gamma_{i} r_{0}\right) J_{l+1}\left(\eta r_{0}\right)\right)\left.\right|_{r_{0}=a_{i-1}} ^{r_{0}=a_{i}}\right\}\right]
\end{aligned}
$$

is a summation of the layers where $k n_{i}<\beta$. Furthermore, 


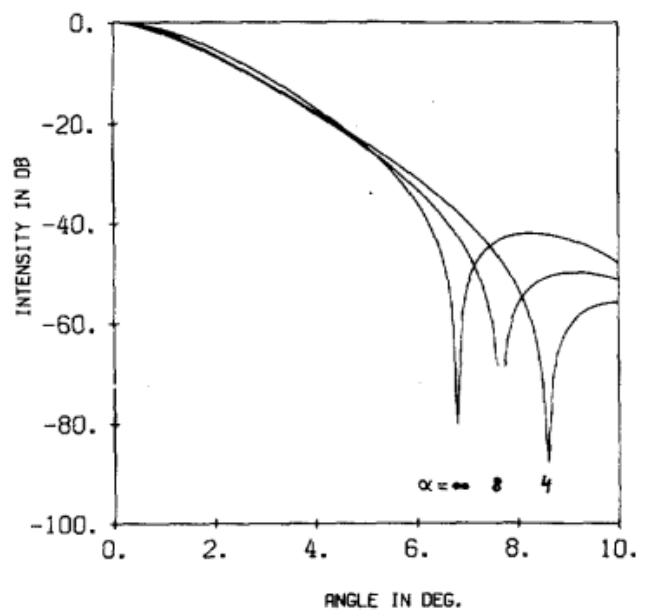

Fig. 3. Intensity of the far field $\left|E_{f}\right|^{2}$ from a single-mode fiber with core radius equal to $5 \mu \mathrm{m}$ and normalized frequency [13] equal to 2.4 versus the angle $\theta$. The profile parameter $\alpha$ is shown to be $\alpha=4$, $8, \infty$.

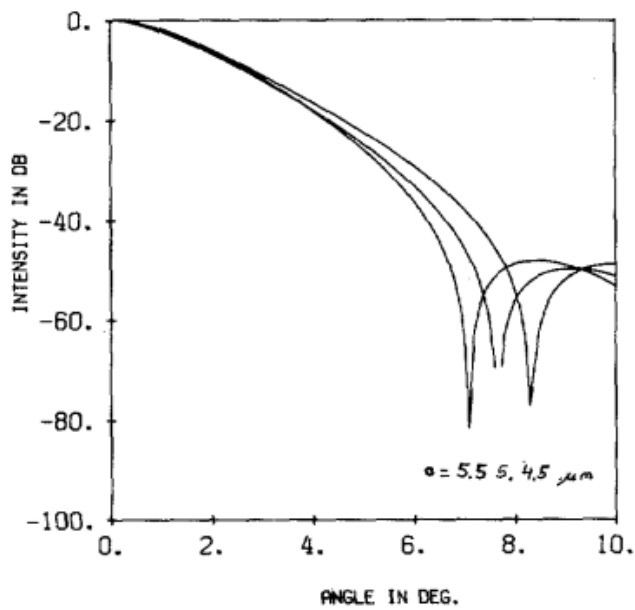

Fig. 4. Intensity of the far field $\left|E_{f}\right|^{2}$ from a single-mode fiber with the profile parameter $\alpha$ equal to 8 and the core radius $a$ as parameter versus the angle $\theta$. The values of $a$ are shown to be 4.5, 5.0, and $5.5 \mu \mathrm{m}$. The normalized frequency is 2.4 when $a$ is equal $5.0 \mu \mathrm{m}$.

$$
\eta=k \sin \theta \text {. }
$$

In the special case of a step-index fiber, there is only one term in each sum and $B_{1}=A_{2}=0$. By use of each characteristic equation [13] with $l=0$ to replace the modified Bessel functions with Bessel functions, we obtain the same result as in [16].

The intensity of the far field $\left|E_{f}\right|^{2}$ is normalized to be $0 \mathrm{~dB}$, when the angle $\theta$ is zero. In Fig. 3, the far field from an $\alpha$ profile [2] with the $\alpha$ value as a parameter is shown. It can be seen that the variation of $\alpha$ mainly causes changes in the far field for angles near the first zero. In Fig. 4 is shown the far field with the core radius as a parameter, and again there are mainly changes in the far field for angles near the first zero.

\section{CONCLUSION}

Convergence tests of the multilayer method have shown that very precise results are obtained with relatively few layers, i.e., 10 layers. The accuracy on the dispersion is better than 0.2 $\mathrm{ps} / \mathrm{km} \cdot \mathrm{nm})$ in a parabolic profile and probably of the same order of magnitude in other profiles. Together with the advantage of analytical expressions for group delay and far field, a very general and efficient model for fibers with a few modes and arbitrary profiles is established.

\section{ACKNOWLEDGMENT}

The author wishes to acknowledge J. Buus, Electromagnetics Institute, for his helpful suggestions.

\section{REFERENCES}

[1] J. Sakai, K. Kitayama, M. Ikeda, Y. Kato, and T. Kimura, "Design considerations of broad-band dual-mode optical fibers," IEEE Trans. Microwave Theory Tech., vol. MTT-26, pp. 658665 , Oct. 1978

[2] P. Danielsen, J. Buus, and E. Nicolaisen, "Estimation of the index profile in a single-mode fiber by means of farfield and pulse response measurements," J. Opt. Commun., vol. 2, pp. 20-23, 1981.

[3] P.J.B. Clarricoats and K. B. Chen, "Electromagnetic wave propagation along radially inhomogeneous dielectric cylinders," Electron. Lett., vol. 6, pp. 694-695, 1970.

[4] T. Tanaka and S. Suematsu, "An exact analysis of cylindrical fiber with index-distribution by matrix-method and its application to focusing fiber," Trans. IECE Japan, vol. E59, pp.1-8, 1976.

[5] C. Yeh and G. Lindgren, "Computing the propagation characteristics of radially stratified fibers-An efficient method," Appl. Opt., vol. 16, pp. 483-493, 1977.

[6] J. Sakai and T. Kimura, "Bending loss of propagation modes in arbitrary-index profile optical fibers," Appl. Opt., vol. 17, pp. 1499-1506, 1978.

[7] B. Kapany, Optical Waveguides. New York: Academic, 1972, p. 108.

[8] R. Sammut, "Some integrals in the theory of curved optical tibers," Opt. Quantum Electron., vol. 9, p. 539, 1977.

[9] K. Okamoto and T. Okoshi, "Analysis of wave propagation in optical fibers having core with $\alpha$-power refractive index distribution and uniform cladding," IEEE Trans. Microwave Theory Tech., vol. MTT-24, pp. 416-421, 1976.

[10] S. Kobayashi, S. Hibrat. N. Shibata, and T. Izawa, "Refractiveindex dispersion of doped fused silica," in Proc. Int. Conf. Integrated Opt. and Opt. Commun., Tokyo, 1977, pp. 309-312.

[11] W. A. Gambling, H. Matsumura, and C. M. Ragdale, "Mode dispersion, material dispersion and profile dispersion in gradedindex single-mode fibers," Microwaves, Opt., Acoust., vol. 3 , pp. 239-246, 1979.

[12] M. Born and E. Wolf, Principles of Optics. New York: Pergamon, 1970 , p. 370.

[13] D. Gloge, "Weakly guiding fibers," Appl. Opt., vol. 10, pp. 2252-2258, 1971.

[14] M. Abramowitz and I. Stegun, Handbook of Mathematical Functions. New York: Dover, 1970, p. 360.

[15] Y. L. Luke, Integrals of Bessel Functions. New York: McGrawHill, 1962, p. 254.

[16] W. A. Gambling, D. N. Payne, H. Matsumura, and R. B. Dyott, "Determination of core diameter and refractive-index difference of single-mode fibres by observation of the far-field pattern," Microwaves, Opt., Acoust., vol. 1, pp. 13-17, 1976.

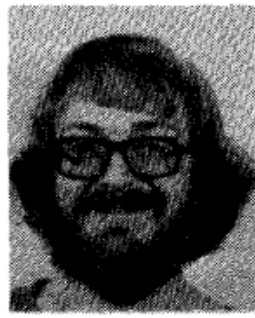

Per L. Danielsen was born in Copenhagen, Denmark, on June 3,1955. He received the M.S. degree in electrical engineering, in 1979 , from the Electromagnetics Institute at the Technical University of Denmark, Lyngby, Denmark, where he is currently working towards the Ph.D. degree.

His research has been on the calculation of the propagation properties in single-mode fibers. He has been working on estimating the index profile by means of a measurement of the far field. 